\title{
Effectiveness of mindfulness-integrated cognitive behavior therapy on anxiety, depression and hope in multiple sclerosis patients: a randomized clinical trial
}

\author{
Sahar Pouyanfard, ${ }^{1}$ (D) Mohsen Mohammadpour, ${ }^{1}$ (D) Ali A. ParviziFard,${ }^{1}$ Kheirollah Sadeghi ${ }^{1}$
}

\begin{abstract}
Introduction: Multiple sclerosis (MS) is a chronic medical condition that attracts particular attention because of the high risks associated with it. MS patients suffer from medical problems, depression, anxiety, and reduced hopefulness. These issues can increase the severity of the disease and treatment resistance and reduce patients' individual and social efficacy. Mindfulness-integrated cognitive behavior therapy (MICBT) is a new approach that is being applied in chronic diseases and can be used in combination with existing treatments. Therefore, the present study investigated the efficacy of MICBT in terms of anxiety, depression, and hope in MS patients.

Methods: A sample of 20 patients with MS was randomly selected at Shafa Hospital in Kerman City, Iran. Patients were then assigned to one of two groups of 10 people using a random number table. The experimental group received MICBT. The control group also received the same therapy after study completion. The assessment tools used in this study included the Beck Depression Inventory-Second Edition (BDI-II), Beck Anxiety Inventory (BAI), and Miller Hope Scale (MHS). Measurements were conducted at three stages: pre-test, post-test, and follow-up. For data analysis, means and standard deviations were calculated and one-way analysis of covariance was conducted using SPSS 24.

Results: Compared with controls, MICBT was effective for reducing depression $(P<0.001, F=72.55)$, anxiety $(P<0.001, F=100.75)$. Additionally, MICBT was effective in improving hope $(P<0.001, F=$ 45.36). Changes were maintained in the follow-up phase.

Conclusion: The MICBT affects depression, anxiety and hope of MS patients. Therefore, mental health professionals can benefit from the results obtained in the present study to reduce depression and anxiety and increase hope in this group of patients.

Clinical trial registration: Iranian Registry of Clinical Trials, IRCT201601030258N4.

Keywords: Group therapy, mindfulness-integrated cognitive-behavioral treatment, multiple sclerosis.
\end{abstract}

\section{Introduction}

Multiple sclerosis (MS) is a complicated, disabling, and immune-related disease, involving the central nervous system (CNS), including the brain and the spinal cord. The etiology of MS remains unidentified; the consensus is that MS is probably caused by either an abnormality of the immune system, or contact with environmental factors (infectious agents), or a combination of both. ${ }^{1}$ Experts recognize $\mathrm{MS}$ as an autoimmune disease; indicating that the immune system mistakenly attacks the body's healthy organs as though they were foreign invaders. MS is among the most prevalent chronic diseases of the CNS and it is a demyelinating disease,

\footnotetext{
${ }^{1}$ Department of Clinical Psychology, Kermanshah University of Medical Sciences, Kermanshah, Iran.

Submitted Jan 06 2019, accepted for publication Jun 262019.

Suggested citation: Pouyanfard S, Mohammadpour M, ParviziFard AA, Sadeghi K. Effectiveness of mindfulness-integrated cognitive behavior therapy on anxiety, depression and hope in multiple sclerosis patients: a randomized clinical trial. Trends Psychiatry Psychother. 2020;42(1):55-63. http://dx.doi.org/10.1590/2237-
} 6089-2018-0105 
affecting motor-sensory functions. ${ }^{2}$ It is estimated that approximately 2-3 million people suffer from MS globally; According to Iran's MS Association, there are 40,000 MS patients in Iran, 9,000 of whom are registered cases. ${ }^{3}$ MS frequently occurs in the 20-40 years age group ${ }^{4}$; i.e. the age associated with greatest family and social responsibilities and with reproduction. ${ }^{5}$ Due to its nature, numerous mental symptoms are correlated with MS. Depression and anxiety are the most prevalent mental symptoms associated with MS and are observed in $48 \%$ of MS sufferers during the year following diagnosis of their disease. ${ }^{6}$ Depression symptoms are the most common mental symptoms in this group. MS can lead to depression in 3 different ways, as follows: 1) various behavioral changes can occur if MS affects the areas of the brain involved in emotional expression and emotion control. 2) Endocrine or immune system changes caused by MS can lead to depression. 3) The adverse effects of some medications can cause depression in this population. Regardless of how depression is triggered, it is inevitably associated with reduced hope in relation to life. ${ }^{7}$

Untreated depression in patients diagnosed with a physical illness can lead to symptoms such as magnifying and exacerbating physical symptoms, excessive referral to medical settings and use of healthcare, increased treatment costs, involvement in high-risk behaviors, and even suicide. ${ }^{8}$ Anxiety is another mental problem in this group. Anxiety has been overlooked in MS patients, despite its disabling consequences. ${ }^{6}$ Moreover, persistent anxiety may exacerbate the disease and lead to cardiovascular disorders. ${ }^{9}$ Awareness of the degenerative nature of the disease causes anxiety in patients. ${ }^{10}$ Recent studies indicate that the prevalence rate of anxiety in MS patients is $37 \% .{ }^{11}$ Additionally, MS patients recognize anxiety as the most disabling sign of their disease. ${ }^{12}$ Hopefulness is impacted negatively in MS, like any other chronic diseases. ${ }^{13}$ Tennen and Affleck (quoted by Bijari et al.) argued that hope plays an essential role in improvement of illness, since it creates positive thoughts about life and an increased tendency to identify the positive aspects of harmful conditions. ${ }^{14}$ MS reduces hopefulness in sufferers, to a level equivalent to 10 years older than their actual age. ${ }^{15}$

In response to the abovementioned issues, different treatment methods, including pharmacotherapy and cognitive behavioral therapy (CBT) have been used to attempt to improve the mental symptoms of MS. However, due to various reasons, such as the adverse effects of medications, the probability of recurrence of symptoms, the ineffectiveness of CBT, and the nonimprovement of emotion regulation skills in these patients, the present study considers a more effective treatment approach that minimizes such problems. ${ }^{15}$ Effective individual and group-based treatments are available for these conditions. It is believed that group treatment is more effective for most mental illnesses. This is because people review how they respond to the community. Furthermore, group therapy is a process leading to improvements in cognitive, emotional and behavioral aspects. Patients feel empowered in the group, which elevates their self-confidence. ${ }^{16}$

Prior research suggests that a new generation of cognitive-behavioral therapies, known as mindfulness and acceptance-based interventions, are more effective for patients with chronic physical illnesses. Mindfulnessintegrated cognitive behavior therapy (MICBT) is a structured approach, integrating mindfulness meditation with the main principals of CBT. This integration is intended to train clients to regulate their emotions and attention, to use these skills when the pathology is triggered, and to understand in which of the mental pathologies they are involved. ${ }^{17}$ MICBT consists of a set of evidence-based techniques to enhance self-awareness, self-control, and self-efficacy in different areas of life. During this course, a person learns how to identify and modify destructive or disturbing intellectual patterns that have a negative impact on his/her behavior.

The weekly schedule of MICBT is flexible and compatible with individual and group conditions. A literature review reveals that group MICBT increases clients' commitment to treatment. ${ }^{16,17}$ Several studies have also reported that MICBT is an effective and appropriate intervention for MS patients and is effective for improving psychological well-being, quality of life, ${ }^{18}$ and depression and alcohol abuse, ${ }^{19}$ and for increasing hope. However, further studies are required to introduce this treatment as an effective therapy for MS. Therefore, the present study aimed to answer the following questions: Does MICBT cause a significant reduction in depression in people with MS? Does MICBT cause a significant reduction in anxiety in MS patients? Does MICBT significantly increase hope in patients with MS?

\section{Materials and methods}

\section{Study design}

This was a repeated measure clinical trial study with a control group. The study consisted of one experimental group (that received MICBT), and one control group.

\section{Statistical population and sampling method}

The statistical population of the current study included all MS patients who were referred to Shafa 
Hospital in Kerman City, Iran, from February 21 to April 20,2018 , and diagnosed with MS by a neurologist.

\section{Sample size}

The sample size was calculated on the basis of prior research, using the formula shown below. ${ }^{20}$ The sample size needed in each group was estimated as about 8 people, for a $95 \%$ confidence interval (CI) and a $20 \%$ type II error. However, considering probable losses from samples, this number was increased to 10 , for a total sample size of 20 patients. A total of 41 patients were initially invited to participate in the study; but some of them were excluded because they did not meeting the inclusion criteria for the study. There were no losses from the sample at the post-test or follow-up stages of the present study.

$$
n=\frac{\left(Z_{1-\alpha / 2}+Z_{1-\beta}\right)^{2}\left(S D_{1}+S D_{2}\right)^{2}}{d^{2}}
$$

\section{Inclusion and exclusion criteria}

The inclusion criteria for the study were a diagnosis of relapsing-remitting MS by a neurologist; confirmation of MS diagnosis by diagnostic procedures (e.g. magnetic resonance imaging, etc.); patient aware of MS disease for 1 year prior to enrollment; age in the range of 18-50 years; signature of written informed consent form for participation in the study; and education to at least the 9th grade (to be able to complete the questionnaires). Exclusion criteria comprised comorbid physical illnesses; psychosis and/or delirium during the course of treatment; severe arousals (e.g. extreme anger, uncontrolled restlessness, or impulsiveness); and receiving other psychological treatments during the course of the study.

\section{Interventions and randomization}

Each study participant was coded with a number after enrolment. The sample was divided into two groups (10 patients per group) with a computerized random number generator, using the permuted block randomization method. Control group members also received MICBT after study completion.

\section{Study tools}

The demographic data questionnaire

This was a questionnaire constructed by the researchers, covering age, gender, and educational level.

Clinical interview, screening by therapist (41)

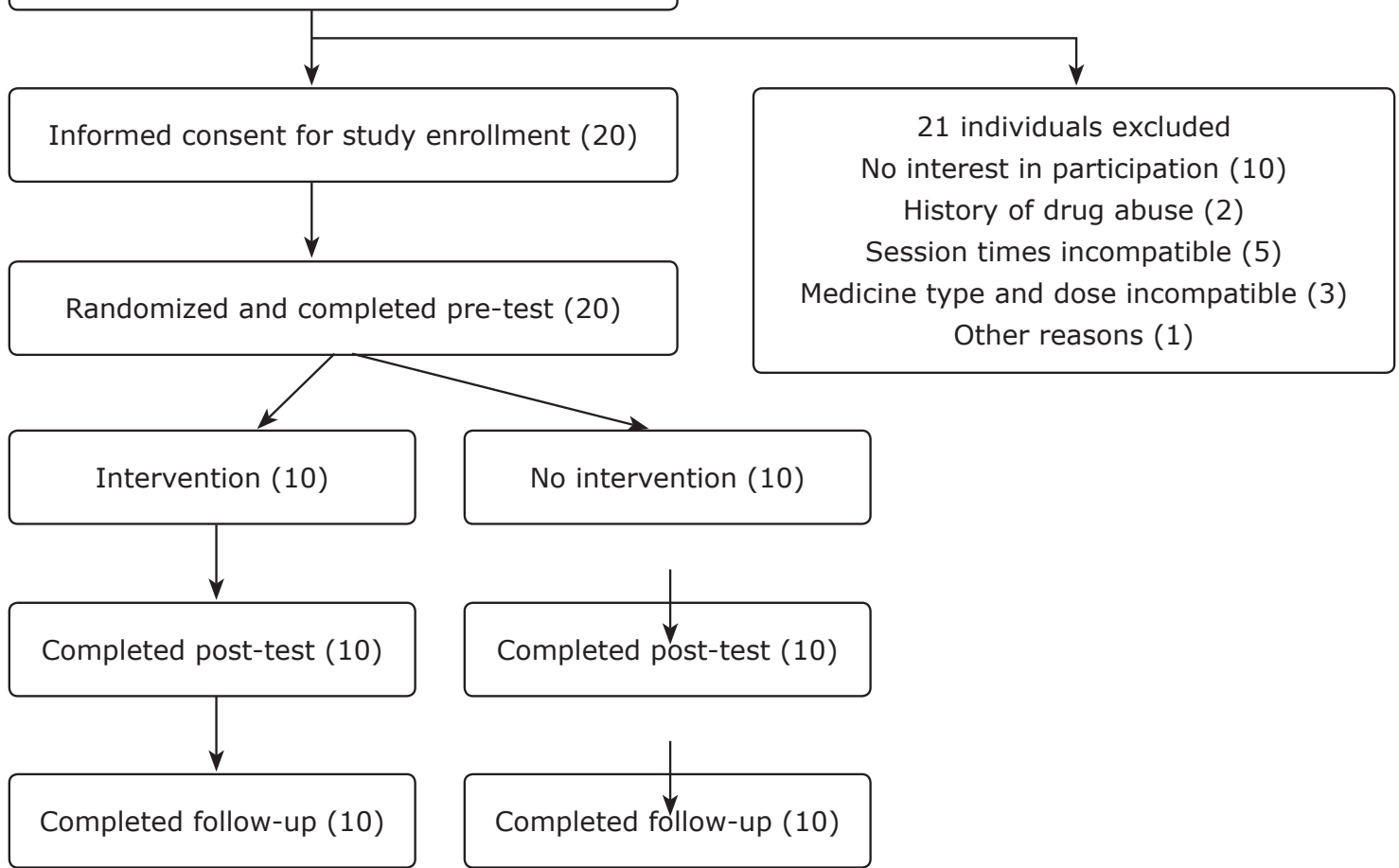

Figure 1 - Flowchart illustrating the study. 
Beck Depression Inventory-Second Edition (BDI-II)

This questionnaire was developed by Beck to measure the severity of depression (1963) and was revised in 1994. This inventory includes 21 items, each scored from 0 to 3 . The highest score achievable on this questionnaire is 63 . Each item on the questionnaire measures one symptom of depression. Its retest reliability is reported as 0.48 to 0.86 , with a mean score of $0.86 .{ }^{21}$ Ghassemzadeh et al. reported an alpha coefficient of 0.87 and a test-retest coefficient of 0.74 for this tool and found a correlation with the first edition of BDI of $93.0 .^{22}$

\section{Beck Anxiety Inventory (BAI)}

This is a 21 -item self-report scale for measuring anxiety. The questionnaire was developed to cover 21 anxiety symptoms and each question is scored from 0 to 3 on a Likert-type scale. Higher scores indicate more severe anxiety. BAI generally targets the physiological aspects of anxiety. Three items address anxious mood, three items address specific phobias, and the rest of the questions evaluate the autonomous symptoms of hyperactivity and motion tension of anxiety. Beck and Clark (1988) reported the internal consistency of the scale as 0.93 and retest reliability of 0.75 . Previous studies demonstrated the high reliability and internal consistency of this tool ( 0.92 ; data correlation: between 0.3 and 0.76$).{ }^{23}$ A survey of 1,513 men and women from various demographic groups (age, gender) in Tehran approved the reliability and validity of the BAI for the Iranian population (reliability:0.72, validity: 0.83, internal consistency: 0.92). ${ }^{24}$

\section{Miller Hope Scale (MHS)}

The Miller Hope Scale (MHS) is a 40-item 5-point Likert-type scale developed to measure hope in adults. The scale was pretested on 75 subjects, then refined and evaluated using 522 healthy students. The score range is 40-200 with a higher score indicating a higher level of hope. Twelve of the items on this scale are formed with negative clauses, which are reverse-scored in the assessment. The MHS showed relatively high construct and concurrent validity compared to other wellestablished tools. In factor analysis, Miller found that the items could be grouped in three components: Satisfaction with self, others, and life; Avoidance of hope threats; and Anticipation of a future. ${ }^{25}$ The internal consistency alpha was reported as 0.81 . Construct validity was established by calculating the correlations of the Herth Hope Index with the MHS $(r=0.43)$, and the MHS with the Herth Hope Index $(r=0.62)$ at $<0.001$ significance level. ${ }^{26}$

\section{Intervention procedure}

The authorities of Shafa Hospital in Kerman were initially requested to explain the purpose of the research to MS patients and introduce them to the researchers. Then, an independent evaluator conducted initial assessments of patients to determine whether they met the inclusion and exclusion criteria of the study. Individuals who met the inclusion criteria for the study and provided an informed consent form were enrolled on the project. The size of the sample analyzed in the present study was 20 people, who were randomly divided into two groups, an experimental group and a control group. The control group received their usual treatment, while the intervention group participated in MICBT sessions as well as drug therapy. A treatment program of 8 two-hour sessions was provided to the experimental group. Post-test measurements were conducted after the intervention and the follow-up stage was conducted two months after completion of treatment. The intervention provided in this study was extracted from a current book on practicing MICBT (covering the principles and method of implementation), written by Bruno Cayoun. ${ }^{15}$ The intervention was conducted by an MSc in clinical psychology (first author) who had undertaken specialized training in this area under supervision of a PhD in Clinical Psychology.

Mindfulness-integrated cognitive behavior therapy includes internalization and externalization skills, and consists of 4 steps, as follows: 1 ) the individual stage 2 ) the exposure stage 3 ) the interpersonal stage, and 4 ) the stage of creating love and kindness for one's self and others; it comprises 8 treatment sessions. At the first meeting, MICBT was introduced to the participants, explaining that it aims to create a commitment to daily practices. The principles of mindfulness were explained to the patients and the treatment was clearly explained to them. In the second session, the most important mechanisms were introduced. The third session provided study participants with visceral insight and intellectual equilibrium. The second phase was introduced in the fourth session. In the fifth session, the second stage was continued at a more challenging level and the third stage was introduced. The sixth session consisted of introduction of assertiveness skills and role-playing as a means of encounter. In the seventh session, the fourth stage was introduced and at the eighth session, the fourth phase was consolidated and the entire intervention program was reviewed. ${ }^{15}$ Table 1 provides further details of the contents of the treatment sessions. 


\section{Data analysis}

The data obtained were analyzed using SPSS 24. Mean scores and standard deviations were calculated and one-way analysis of covariance (ANCOVA) was conducted.

\section{Ethical considerations}

Prior to initiating the study, a meeting was held to explain the project to patients. At this meeting, ethical issues were presented and the research was explained to the patients. Then, everyone completed informed consent forms and the study participants were assured that their results and identity would be kept confidential, and that under no circumstances would their health information be provided to any person except the medical staff. The study was approved by the Research Ethics Committee at Kermanshah University of Medical Sciences (ethics code: kums.rec.1396.616). This study was also registered on the Iranian Registry of Clinical Trials (code: IRCT201601030258N4).

\section{Results}

Descriptive findings of research: Table 2 lists the demographic variables for the sample participants.

As per Table 2, the number of participants in the study sample was 20 . There were 8 male participants ( 4 in the experimental group and 4 in the control group), and 12 female participants ( 6 in the experimental group and 6 in the control group). The educational level of the samples was as follows: Experimental group: elementary school (1), high school (2), associate degree (1), bachelor degree (4), and master degree (2). Control group: elementary school (1), high school (2), associate degree (0), bachelor degree (5) and master degree (2).

Table 3 lists the means and standard deviations for study variables in the experimental and control groups at the pre-intervention, post-intervention, and follow-up stages. As the results show, the intensity of depression and anxiety significantly decreased in the intervention

Table 1 - The curriculum for MICBT sessions

\begin{tabular}{|c|c|}
\hline Session & Content \\
\hline 1 & $\begin{array}{l}\text { Introductory presentation covering the sessions and rules, an overview of MICBT, the concept and principles of } \\
\text { mindfulness, and mindful breathing }\end{array}$ \\
\hline 2 & $\begin{array}{l}\text { Mindful breathing (continued), an overview of a few MICBT concepts (such as situation, sensory perception, evaluation, } \\
\text { body sensations, and reaction), the internal causes of intrusive thoughts and their modification, part-by-part body } \\
\text { scanning }\end{array}$ \\
\hline 3 & Part-by-part body scanning (continued), explaining about body sensations, informal practice \\
\hline 4 & $\begin{array}{l}\text { Body scanning exercises (continued), introducing some of the CBT components of MICBT (like exposure methods), } \\
\text { encountering unpleasant sensations using SUDS (a scale used for exposure to target events) through bipolar exposure } \\
\text { (imaginary exposure to unpleasant situations) }\end{array}$ \\
\hline 5 & Body scanning exercises (continued), review of SUDS \\
\hline 6 & Body scanning exercises (continued), interpersonal skills, assertiveness and role-playing \\
\hline 7 & Introducing the concepts of compassion and empathy, loving-kindness meditation \\
\hline 8 & Review and evaluation \\
\hline
\end{tabular}

$\mathrm{CBT}=$ cognitive behavioral therapy; $\mathrm{MICBT}=$ mindfulness-integrated cognitive behavior therapy; SUDS = Subjective Units of Distress Scale.

Table 2 - Demographic properties of sample group

\begin{tabular}{lccccccc}
\hline & \multicolumn{2}{c}{ Gender } & \multicolumn{3}{c}{ Educational level } \\
\cline { 2 - 7 } Group & Male & Female & $\begin{array}{c}\text { Elementary } \\
\text { school }\end{array}$ & High school & $\begin{array}{c}\text { Associate } \\
\text { degree }\end{array}$ & $\begin{array}{c}\text { Bachelor } \\
\text { degree }\end{array}$ & $\begin{array}{c}\text { Master } \\
\text { degree }\end{array}$ \\
\hline Experimental & 4 & 6 & 1 & 2 & 1 & 4 & 2 \\
Control & 4 & 6 & 1 & 2 & 0 & 5 & 2 \\
\hline
\end{tabular}


group, more than in the control group. Additionally, hope increased in the intervention group.

\section{Data on the research hypotheses}

To determine whether the data obtained fulfilled the underlying assumptions for use of ANCOVA, they were tested prior to analyzing the hypothesis data. The test results showed that ANCOVA could be conducted. The assumptions examined included the following: normal distribution of data (Kolmogorov-Smirnov and Shapiro Wilk's tests), homogeneity of the variables (Levene's test), lack of irrelative data (box plots), homogeneity of covariance matrices (Box M test), and a linear relationship between the dependent variable and the covariate (linear regression). We examined the following research hypotheses: MICBT significantly decreases depression in the experimental group, compared to the control group; MICBT significantly reduces anxiety in the experimental group, compared to the controls; MICBT significantly increases hope in participants in the experimental group compared to the control group. One-way ANCOVA was used to analyze the results obtained. Table 4 lists the ANCOVA results for the post-test data and Table 5 shows results for the follow-up phase.

Observing the results from Table 3 and examining the mean values for experimental and control groups in post-experimental data from Table 2, it can be concluded that post-intervention, MICBT group therapy was successful for reducing depression and anxiety scores and improving hope scores. The results in Table 4 show that the difference between the two groups in depression $(P<0.001, F=72.55)$, anxiety $(P<0.000$, $F=100.75)$, and hope $(P<0.001, F=45.36)$ were significant at the post-test stage. This can be interpreted as showing that MICBT group therapy is effective at reducing mean scores for depression and anxiety at the post-intervention stage and for improving hope at the same stage.

The results in Table 5 show that the differences between the two groups in depression $(P<0 / 001, P$ $=85.87)$, anxiety $(P<0 / 001, F=89.19)$, and hope $(P<0 / 001, F=88.35)$ were still significant at the follow-up stage.

Table 3 - Mean and standard deviation for anxiety, depression and hope

\begin{tabular}{|c|c|c|c|c|c|c|}
\hline & \multicolumn{3}{|c|}{ Experimental group } & \multicolumn{3}{|c|}{ Control group } \\
\hline & Pre-test & Post-test & Follow-up & Pre-test & Post-test & Follow-up \\
\hline BDI-II & $\begin{array}{l}43 / 25 \\
(3 / 73)\end{array}$ & $\begin{array}{c}24 \\
(6 / 86)\end{array}$ & $\begin{array}{l}25 / 12 \\
(5 / 87)\end{array}$ & $\begin{array}{c}45 / 12 \\
(1 / 8)\end{array}$ & $\begin{array}{l}47 / 37 \\
(1 / 5)\end{array}$ & $\begin{array}{l}46 / 5 \\
(1 / 6)\end{array}$ \\
\hline BAI & $\begin{array}{l}51 / 37 \\
(4 / 59)\end{array}$ & $\begin{array}{l}26 / 75 \\
(5 / 03)\end{array}$ & $\begin{array}{l}28 / 12 \\
(4 / 25)\end{array}$ & $\begin{array}{l}50 / 37 \\
(4 / 06)\end{array}$ & $\begin{array}{l}49 / 75 \\
(4 / 62)\end{array}$ & $\begin{array}{c}48 / 75 \\
(5)\end{array}$ \\
\hline MHS & $\begin{array}{l}107 / 37 \\
(3 / 66)\end{array}$ & $\begin{array}{c}168 / 37 \\
(21)\end{array}$ & $\begin{array}{c}166 \\
(21 / 58)\end{array}$ & $\begin{array}{c}108 \\
(6 / 62)\end{array}$ & $\begin{array}{c}112 / 12 \\
(7 / 75)\end{array}$ & $\begin{array}{l}114 / 5 \\
(9 / 62)\end{array}$ \\
\hline
\end{tabular}

Data presented as mean (standard deviation).

BAI = Beck Anxiety Inventory; BDI-II = Beck Depression Inventory-Second Edition; MHS = Miller Hope Scale; SD = standard deviation.

Table 4 - Analysis of covariance results for depression, anxiety, and hope scores from the post-intervention stage (between groups)

\begin{tabular}{lcccccc}
\hline & SS & df & MS & F & Sig. & ES \\
\hline BDI-II & $9,121 / 57$ & 1 & $9,121 / 57$ & $72 / 55$ & $0 / 001$ & $0 / 58$ \\
BAI & $2,162 / 41$ & 1 & $2,162 / 41$ & $100 / 75$ & $0 / 001$ & $0 / 62$ \\
MHS & $1,245 / 19$ & 1 & $1,245 / 19$ & $45 / 36$ & $0 / 001$ & $0 / 61$ \\
\hline
\end{tabular}

$\mathrm{BAI}=$ Beck Anxiety Inventory; BDI-II = Beck Depression Inventory-Second Edition; MHS = Miller Hope Scale; df = degrees of freedom; ES = Eta squared; MS $=$ mean square; $\mathrm{SS}=$ sum of squares.

Table 5 - Analysis of covariance results for depression, anxiety and hope scores in follow-up phase

\begin{tabular}{lccccc}
\hline & SS & df & MS & F & Sig. \\
\hline BDI-II & $1,671 / 76$ & 1 & $1,671 / 76$ & $85 / 87$ & $0 / 001$ \\
BAI & $1,746 / 1$ & 1 & $1,746 / 1$ & $89 / 19$ & $0 / 001$ \\
MHS & $10,787 / 97$ & 1 & $10,787 / 97$ & $88 / 35$ & $0 / 60$ \\
\hline
\end{tabular}

BAI = Beck Anxiety Inventory; BDI-II = Beck Depression Inventory-Second Edition; MHS = Miller Hope Scale; df = degrees of freedom; ES = Eta squared; MS $=$ mean square; $\mathrm{SS}=$ sum of squares.

60 - Trends Psychiatry Psychother. 2020;42(1) 


\section{Discussion}

The data obtained suggest that MICBT significantly decreased depression in the experimental group, compared to controls, and that its impact was sustained to the follow-up stage. These findings are consistent with other studies of the effectiveness of MICBT. ${ }^{20,27-30}$ This could be because mindfulness-based exercises assist MS patients to consider their thoughts as mental events. In other words, mindfulness helped the subjects to acquire the ability to observe their disturbing thoughts and be aware of them, rather than being influenced by them and considering them to be real and definitive, and therefore the mindfulness practices reduced the effect of depressive thoughts on the subjects studied.

Moreover, MICBT uses mindfulness exercises to focus on breathing as a means of living in the present moment, which can reduce concerns related to the past and disturbing worries and thoughts related to the future. According to Teasdale, Segal, and Didona, ${ }^{31}$ mindfulness improves depression by reducing and stopping the negative loops of thought and rumination, teaching people to maintain a distance from thoughts, teaching flexibility of attention, and mental empowerment. Furthermore, along with mindfulness practices, assertiveness training, and role-playing skills as a means of confrontation increased the abilities of the people in the experimental group to be prepared for taking actions and increased their willingness and capability to act, resulting in reductions in the reluctance and lack of desire for taking action that are signs of depression. Additionally, assertiveness training can reduce depression by improving decision-making ability, ${ }^{32}$ experiencing positive emotions, and increasing self-esteem. ${ }^{33}$

The present study also revealed that MICBT significantly reduced anxiety in the experimental group, compared with controls, and that this effect was sustained until the follow-up stage. These findings are consistent with other studies. ${ }^{18,20,29}$ In explaining this finding, it can be argued that MS with its debilitating, chronic, and unpredictable characteristics imposes many challenges on patients' lives. Mindfulness and its training modify feelings without judgments, increase awareness of bio-psychological feelings, and help subjects to clearly experience and accept emotions and physical phenomena, as they occur. ${ }^{15,27}$ In other words, in the experimental group, by providing the ability to clearly recognize emotions, thoughts, and physical conditions without subjective judgments about them, mindfulness reduced disturbing and distracting thoughts that envisage excessively undesirable and disturbing conditions and cause anxiety. Additionally, as confronting measures, assertiveness training and role-playing could have improved preparedness for copying with disease-related changes and life, and reduced feelings of incapability and also could have improved the self-confidence of experimental group members for coping with undesirable conditions. Moreover, cognitive errors (catastrophizing, labeling, negative predicting, etc.) were reduced in the experimental group, which in turn improved the symptoms of anxiety. ${ }^{15}$

The data obtained also demonstrated that MICBT significantly increased hope among the participants in the experimental group, compared with the controls, and that this effect was also sustained to the follow-up stage. These findings are in agreement with previous work. ${ }^{19,34}$ In explaining this finding, it can be argued that MICBT teachings help patients to reduce their stress, change their conditions, strengthen their life tendency, cope with depression, frustration, and despair, and, ultimately, to gain optimism and trust. Mindfulness also helps individuals identify situations that cause anxiety and stress, develop a better knowledge of themselves and their thoughts, recognize their strengths and flaws, and achieve better and higher awareness regarding their emotions, thoughts, physical states, and illness. They may then be able to find coping strategies to manage these situations, thereby creating hope in their lives. Furthermore, assertiveness training and role-play also help to build confidence and better readiness to act in the future, which also increases hope in this group. Overall, the results obtained in this intervention suggest that the MICBT was effective for MS patients. MS causes fatigue and illness, and the number of research questions was high; thus, the pre-test-post-test results may have been influenced by these issues. Moreover, the same tool was applied for pre-test, post-test, and followup stages; therefore, familiarity with the test in the pre-test step may have affected the post-test and follow-up results. It is suggested that psychologists and counselors use MICBT as a therapeutic strategy to reduce anxiety and depression and increase hope among MS patients.

Several limitations of this investigation need to be mentioned. One of the study limitations was the lack of theoretical background for the study, because of the novelty of the intervention. The present study's 2-month follow-up period may not be sufficient to assess longterm maintenance of treatment gains. Future studies are recommended to perform long-term follow-up assessments in order to evaluate the effects of MICBT on MS patients. 


\section{Conclusion}

This study provided further evidence for the effectiveness of MICBT. MS patients face a variety of stresses in everyday life, in addition to the stress related to their disease. One of the most stressful aspects of this disease is uncertainty with relation to the future. Coping with MS can be very challenging and the consequences of diagnosing the disease in people can lead to various emotions such as sorrow, hopelessness, anger, anxiety, depression or distrust. Hence, mindfulness therapy can be mastered through techniques such as experiencing the present, deep breathing, relaxation, and better understanding of thoughts, better understanding of emotions, and physical states, which, in turn, will help the affected person to achieve acceptance of the illness and enact behavioral changes for better selfcare. The patient can thus become better prepared to identify appropriate coping strategies to manage challenging situations using techniques such as roleplaying and assertiveness to reduce anxiety and depression and increase hope. Therefore, with regards to the effectiveness of MICBT for reducing anxiety and depression and increasing hope, and in view of the increasing incidence of MS in the country, the results of this study may be beneficial to treatment programs and services for MS patients aiming to increase hope and reduce anxiety and depression among them.

\section{Disclosure}

No conflicts of interest declared concerning the publication of this article.

\section{References}

1. Goetz CG. Textbook of Clinical Neurology. Amsterdam: Elsevier Health Sciences; 2007.

2. MCcabe MP. Mood and self-esteem of persons with multiple sclerosis following an exacerbation. J Psychosom Res. 2005; 59:161-6.

3. Taghizadeh ME, Miralaei MS. The study of the effectiveness of group spirituality therapy on resilience of women with multiple sclerosis, city of Isfahan. Health Psychol. 2013;2:82-102. [Persian]

4. Aghabagheri $\mathrm{H}$, Mirzaeian B, Mohammad Khani $\mathrm{P}$, Omrani S. The effectiveness of the mentally cognitive therapy group (MBCT) on depression in multiple sclerosis patients. Thought Behav Clin Psychol. 2012;6:75-82. [Persian]

5. Kinney MR. Quality of life research: rigor or rigor mortis. Cardiovasc Nurs. 1995;31:25-8.

6. Mitchell AJ, Benito-León J, González JM, Rivera-Navarro J. Quality of life and its assessment in multiple sclerosis: integrating physical and psychological components of wellbeing. Lancet Neurol. 2005;4:556-66.

7. Ghara ZF, Aliakbari DM, Alipour A, Mohtashami T. Efficacy of group logo therapy in the perceived stress and life expectancy in MS patients. Res Psychol Health. 2013;6:12-20. [Persian]
8. Mohr DC, Boudewyn AC, Goodkin DE, Bostrom A, Epstein L. Comparative outcomes for individual cognitive-behavior therapy, supportive-expressive group psychotherapy, and sertraline for the treatment of depression in multiple sclerosis. J Consult Clin Psychol. 2001;69:942-9.

9. Potagas C, Mitsonis C, Watier L, Dellatolas G, Retziou A Mitropoulos $\mathrm{P}$, et al. Influence of anxiety and reported stressful life events on relapses in multiple sclerosis: a prospective study. Mult Scler. 2008;14:1262-8.

10. Feinstein A, O'Connor P, Gray $T$, Feinstein $\mathrm{K}$. The effects of anxiety on psychiatric morbidity in patients with multiple sclerosis. Mult Scler. 1999;5:323-6.

11. Korostil M, Feinstein A. Anxiety disorders and their clinica correlates in multiple sclerosis patients. Mult Scler. 2007;13:6772.

12. Stenager $E$, Knudsen L, Jensen K. Multiple sclerosis: correlation of anxiety, physical impairment and cognitive dysfunction. Ital J Neurol Sci. 1994;15:97-101.

13. Dennison L, Moss-Morris R, Chalder T. A review of psychological correlates of adjustment in patients with multiple sclerosis. Clin Psychol Rev. 2009;29:141-53.

14. Longi D, Kasper D, Jameson L, Faucl A, Hauser A, Loscalzo J, eds. Harrison's principles of Internal Medicine. 20th ed. New York: McGraw Hill; 2018. p. 3198-205.

15. Cayoun B. Mindfulness-integrated CBT principles and practice. 2nd ed. Tehran: University of Tehran Press; 2011. 173-4. Translated from Persian by Khodayarifard M, Mohammadi Hasel K, Didehdar M.

16. Ezhumalai S, Muralidhar D, Dhanasekarapandian R, Nikketha BS. Group interventions. Indian J Psychiatry. 2018;60:514-21.

17. Cayoun BA. Mindfulness-integrated CBT for well-being and personal growth: four steps to enhance inner calm, selfconfidence and relationships. Hoboken: John Wiley \& Sons; 2014. p. 269-71.

18. Fiest KM, Bernstein CN, Walker JR, Graff LA, Hitchon CA, Peschken $C A$, et al. Systematic review of interventions for depression and anxiety in persons with inflammatory bowel disease. BMC Res Notes. 2016;9:404.

19. Baker AL, Kavanagh DJ, Kay-Lambkin FJ, Hunt SA, Lewin TJ, Carr VJ, et al. Randomized controlled trial of MICBT for co-existing alcohol misuse and depression: outcomes to 36-months. J Subst Abuse Treat. 2014;46:281-90.

20. Bahrani S, Zargar F, Yousefipour G, Akbari H. The effectiveness of mindfulness-integrated cognitive behavior therapy on depression, anxiety, and stress in females with multiple sclerosis: a single blind randomized controlled trial. Iran Red Crescent Med J. 2017; 19:e44566.

21. Beck AT, Steer RA, Brown GK. Manual for the Beck Depression Inventory-II. San Antonio: Psychological Corporation; 1996.

22. Ghassemzadeh $\mathrm{H}$, Mojtabai R, Karamghadiri N, Ebrahimkhani N. Psychometric properties of a Persian $\square$ language version of the Beck Depression Inventory--Second Edition: BDI $\square$ II $\square$ PERSIAN. Depress Anxiety. 2005;21:185-92.

23. Beck AT, Epstein N, Brown G, Steer RA. An inventory for measuring clinical anxiety: psychometric properties. J Consult Clin Psychol. 1988;56:893-7.

24. Kaviani HH, Mousavi AS. Psychometric properties of the Persian version of Beck Anxiety Inventory (BAI). Tehran Univ Med J. 2008;66:136-40.

25. Miller JF, Powers MJ. Development of an instrument to measure hope. Nurs Res. 1988;37:6-10.

26. Abdi N, Asadi Lari M. Standardization of three hope scales, as possible measures at the end of life, in Iranian population. Intern J Cancer Prev. 2011;4:71-7.

27. Simpson R, Booth J, Lawrence M, Byrne S, Mair F, Mercer $\mathrm{S}$. Mindfulness based interventions in multiple sclerosis--a systematic review. BMC Neurol. 2014;14:15.

28. Hoseyni S. The effect of group cognitive therapy in reducing depression and increasing mental health of patients with multiple sclerosis. Tehran: Alzahra University; 2005.

29. Muñoz San Jose A, Oreja-Guevara C, Cebolla Lorenzo SC, Carrillo Notario L, Rodríguez Vega B, Bayón Pérez CB. Psychotherapeutic and psychosocial interventions for managing stress in multiple sclerosis: the contribution of mindfulness-based interventions. Neurologia. 2016;31:113-20.

30. Yazdanimehr $R$, Omidi A, Sadat $Z$, Akbari $H$. The effect of mindfulness-integrated cognitive behavior therapy on depression and anxiety among pregnant women: a randomized clinical trial. J Caring Sci. 2016;5:195. 
31. Didonna F. Clinical handbook of mindfulness. New York: Springer; 2009.

32. Tomaka J, Palacios R, Schneider KT, Colotla M, Concha JB, Herrald MM. Assertiveness predicts threat and challenge reactions to potential stress among women. J Pers Soc Psychol. 1999; 76:100821.

33. Landoni MG, Giordano MT, Guidetti GP. Group psychotherapy experiences for people with multiple sclerosis and psychological support for families. J Neurovirol. 2000;6 Suppl 2:S168-71.

34. Bagheri H, Mirzaeayan B, MohammadGhani P, Omrani S. The efficacy of mindfulness-based cognitive therapy (MBCT) on the reduction of depression in patients with multiple sclerosis (MS). Thought Behav. 2012;6:75-82.

\section{Correspondence:}

Ali A. ParviziFard

Department of Clinical Psychology

Kermanshah University of Medical Sciences,

7617196171 - Kermanshah - Iran

Tel: +98918 3308692

E-mail:parvizia@yahoo.com 


\section{http:/ / dx.doi.org/10.1590/2237-6089-2020-4203}

The authors of the article entitled "Effectiveness of mindfulness-integrated cognitive behavior therapy on anxiety, depression and hope in multiple sclerosis patients: a randomized clinical trial" (doi: https://doi.org/10.1590/22376089-2018-0105), published in Trends in Psychiatry and Psychotherapy, volume 42, issue 1, pages 55-63, would like to correct the order in which the authors are presented, more specifically the positions of the second and third authors. Below we present the correct version of the author byline:

Sahar Pouyanfard, Mohsen Mohammadpour, Ali A. ParviziFard, Kheirollah Sadeghi ${ }^{1}$

Trends Psychiatry Psychother. 2020;00(0):000. 